\title{
Estudo da estabilidade acelerada de formulações contendo cetoconazol xampu a $2 \%$
}

\author{
Amanda Leitão Gindri*, Lucas Leitão Gindri**, Letiele Bruck de \\ Souza***, Marcos Roberto dos Santos*, Luciane Varini Laporta****
}

\begin{abstract}
Resumo: 0 objetivo deste estudo foi avaliar a estabilidade acelerada de duas formulações magistrais de cetoconazol xampu, determinar seu prazo de validade e comparar estes resultados com as formulações industrializadas. Utilizou-se o medicamento referência, um genérico e um similar, além de duas formulações manipuladas, todas contendo cetoconazol na concentração de $2 \%$. As amostras foram armazenadas em $4{ }^{\circ} \mathrm{C}, 25^{\circ} \mathrm{C}$ e $40{ }^{\circ} \mathrm{C}+70 \%$ UR por 180 dias e avaliadas em relação a cor, odor, densidade, viscosidade e teor, conforme a Farmacopéia Brasileira. Todos os produtos foram aprovados nos testes de identificação, volume médio e doseamento. As formulações comerciais praticamente não sofreram alterações de cor e odor quando armazenadas a $4{ }^{\circ} \mathrm{C}$ e $25^{\circ} \mathrm{C}$, já os medicamentos manipulados mantiveram-se estáveis a temperatura de $4{ }^{\circ} \mathrm{C}$, mas quando armazenadas a $25{ }^{\circ} \mathrm{C}$ sofreram pequenas alterações de cor, próximo aos 180 dias. Não houve alterações consideráveis de $\mathrm{pH}$ e densidade, em todas as amostras. Foi impossivel determinar a faixa ideal de viscosidade. Os medicamentos comerciais e a formulação 1 mostraram boa estabilidade, sendo possível conseguir um prazo de validade provisório de 24 meses.
\end{abstract}

Descritores: Estabilidade cetoconazol, Produtos Manipulados, Xampu.

\section{Accelerated stability study of shampoo formulations containing ketoconazole $2 \%$}

\begin{abstract}
This study had the objective to evaluate the stability of two accelerated magistral formulations of ketoconazole shampoo, determine its validity and to compare the results with the formulations industrialized. Was used the drug reference, a generic and a similar, besides the manipulated formulations, all containing ketoconazole at a concentration of $2 \%$. The samples were stored at $4{ }^{\circ} \mathrm{C}, 25{ }^{\circ} \mathrm{C}$ and $40{ }^{\circ} \mathrm{C}+70 \%$ UR, for 180 days and evaluated for color, odor, density, viscosity and dosage. All products have passed the tests for identification, dosage and medium volume. The commercial formulations virtually unchanged in color and odor when stored at $4{ }^{\circ} \mathrm{C}$ and $25^{\circ} \mathrm{C}$, and manipulated formulations remained stable at a temperature of $4{ }^{\circ} \mathrm{C}$, but when stored at $25^{\circ} \mathrm{C}$ began to experience minor changes in color, close to 180 days. No significant changes on $\mathrm{pH}$ and density for all samples were observed. Was impossible to determine the optimal range of viscosity. The commercial medicines and the formulation 1 showed good stability, being possible to estimate the expiration time of 24 months.
\end{abstract}

Descriptors: Ketoconazole stability, Manipulated products, Shampoo.

*Mestre em Ciências Farmacêuticas pela Universidade Federal de Santa Maria (UFSM), Santa Maria, RS, Brasil.

**Graduado em Farmácia com formação Generalista pelo Centro Universitário Franciscano (UNIFRA), Santa Maria, RS, Brasil.

${ }^{* * *}$ Graduada em Biologia pela Universidade da Região da Campanha (URCAMP), Bagé, RS, Brasil. Mestranda em Agrobiologia pela Universidade Federal de Santa Maria (UFSM), Santa Maria, RS, Brasil.

****Mestre em Ciência e Tecnologia Farmacêuticas pela Universidade Federal de Santa Maria (UFSM), Santa Maria, RS, Brasil. 


\section{Introdução}

Cetoconazol é um antifúngico da classe dos imidazólicos, sendo amplamente utilizado no tratamento de infecções graves. Tem demonstrado efetividade contra infecções ginecológicas, pneumonia fúngica, infecções de pele e mucosas, septicemias, profilaxia de micoses em pacientes imunodeprimidos e outras infecções abdominais ${ }^{1}$.

Como os demais derivados imidazólicos antifúngicos, o cetoconazol exerce ação fungicida por alterar a permeabilidade da membrana citoplasmática dos fungos sensíveis, que passam a perder cátions, proteínas e outros elementos vitais, ocorrendo, por fim, o rompimento da membrana. Esta ação sobre a membrana é variável de acordo com o fungo e a dose do medicamento, agindo não só sobre as células fúngicas, mas, também, inibindo a síntese de estrogênios e testosterona no homem ${ }^{1,2,3}$.

Existe grande preferência clínica na administração de formulações tópicas com cetoconazol, pois são eficazes em tratamentos de micoses superficiais tanto na pele como em mucosas, sem efeitos adversos significantes ${ }^{4}$.

As farmácias magistrais têm se mostrado uma alternativa quando o paciente necessita de doses personalizadas, o que resultou num crescente aumento da procura por medicamentos manipulados. Outro fator que justifica esse crescimento é o custo desses medicamentos que em geral, são mais baratos que os industrializados ${ }^{5}$.

De uma maneira geral, os medicamentos manipulados sofrem algumas limitações tecnológicas e de controle de qualidade inerente ao processo de produção personalizada. É fundamental ressaltar que diferenças em relação às características físicas e físico-químicas do fármaco e demais componentes da formulação, bem como nos processos de fabricação, podem gerar diferenças na biodisponibilidade podendo comprometer a intercambialidade ${ }^{6}$.

O estudo de estabilidade é parte integrante da garantia da qualidade, tendo por finalidade avaliar o comportamento dos fármacos ou medicamentos que se alteram com 0 tempo, por influência de fatores extrínsecos. Esses estudos possibilitam ainda avaliar possíveis incompatibilidades entre componentes de formulações ou entre estes e materiais de acondicionamento?

Sendo assim, devido à grande facilidade de ocorrer instabilidade em formulações contendo cetoconazol e a necessidade de se determinar o prazo de validade para esses produtos, esse trabalho teve como principais objetivos estudar a estabilidade acelerada de duas formulações magistrais de cetoconazol xampu, determinar seu prazo de validade e comparar os resultados obtidos com as formulações industrializadas.

Saúde (Santa Maria), v.38, n.1, p. 139-149, 2012. Estudo da estabilidade acelerada de formulaçöes contendo cetoconazol xampu a $2 \%$

\section{Materiais e métodos}

Para a realização deste trabalho foi utilizada a substância química de referência (SQR) Cetoconazol, Lote 1033, produzida pela Farmacopéia Brasileira. As amostras de cetoconazol xampu 2\% utilizadas foram: medicamento referência - lote LEL110; medicamento similar lote 806119; medicamento genérico - lote 134973; Formulação manipulada 1, contendo os seguintes excipientes: lauril éter sulfato de sódio; cocoamidopropilbetanina; dietil ácido graxo de coco; solução conservante (nipagin + nipazol) e água; e Formulação manipulada 2, 
contendo os seguintes excipientes: lauril éter sulfato de sódio; cocoamidopropilbetanina; dietil ácido graxo de coco; base perolada texapon, solução conservante (nipagin + nipazol) e água. As duas formulações manipuladas foram obtidas segundo o Formulário Nacional (2001) ${ }^{8}$.

No teste de identificação foram comparados os tempos de retenção do pico principal das soluções amostras, com o pico principal obtido com a substância química de referência $(\mathrm{SQR})$, através do método de doseamento em cromatógrafo líquido.

$\mathrm{Na}$ determinação de volume foram pesados, individualmente, três (3) unidades, removido o conteúdo e lavados os recipientes com água e em seguida com etanol. Secou-se em estufa a 105 oC por 1 hora. Os recipientes foram transferidos para dessecador até atingirem temperatura ambiente. Pesou-se novamente, recolocando as tampas e outras partes correspondentes a cada unidade. A diferença entre as duas pesagens representou 0 peso do conteúdo ${ }^{9}$. $O$ volume foi determinado conforme a equação: $d=m / v$, onde $d$ representa a densidade, $m$ a massa e $v$ o volume.

Para o doseamento foi preparado uma solução amostra, onde transferiu-se 0 equivalente a $20 \mathrm{mg}$ de cetoconazol para um balão volumétrico de $100 \mathrm{~mL}$, adicionou-se 70 $\mathrm{mL}$ de metanol, sonicou-se por 30 minutos, completou-se o volume com metanol e filtrou-se. Transferiu-se $2,5 \mathrm{~mL}$ para um balão volumétrico de $10 \mathrm{~mL}$, adicionou-se $1,5 \mathrm{~mL}$ de metanol e completou-se o volume com água. Também foi preparado uma solução de SQR da mesma forma da solução amostra. A fase móvel utilizada nesta técnica foi uma mistura de acetonitrila - metanol - tetrahidrofurano e tampão fosfato $\mathrm{pH} 7,0$ (39:9,5:1,5:50) e o sistema cromatográfico foi um cromatógrafo provido de detector ultravioleta a $232 \mathrm{~nm}$; coluna de 250 $\mathrm{mm}$ de comprimento e 4,6 mm de diâmetro interno, empacotada com sílica quimicamente ligada a grupo octadecilsilano $\left(\begin{array}{ll}5 & \mathrm{~m}\end{array}\right)$, mantida à temperatura de $40^{\circ} \mathrm{C}$; e o fluxo da fase móvel de 1,5 mL/minuto. Injetou-se, separadamente, $20 \quad \mathrm{~L}$ das soluções padrão e amostra, registrou-se os cromatogramas e mediu-se as áreas dos picos. Calculou-se a quantidade de $\mathrm{C}_{26} \mathrm{H}_{28} \mathrm{Cl}_{2} \mathrm{~N}_{4} \mathrm{O}_{4}$ (cetoconazol) no xampú a partir das respostas obtidas para as soluções padrão e amostra. $O$ desvio padrão relativo das áreas de replicatas dos picos registrados não foi superior a $2 \%$.

O estudo de estabilidade foi conduzido conforme a RE no 1, de 29 de julho de $2005^{10}$. As amostras de cetoconazol xampu foram expostas as temperaturas de geladeira (4 OC), ambiente $(25 \mathrm{oC})$ e estufa ( $40 \mathrm{OC}+70 \% \mathrm{UR})$, durante seis meses e avaliadas no tempo zero, 30 dias, 90 dias e 180 dias, quanto às características físico-químicas, teor, pH, densidade e viscosidade.

As características físico-químicas das amostras foram analisadas em referência a cor e odor, obedecendo-se o seguinte critério: 1 - sem alteração; 2 - pequena alteração; 3 - grande alteração.

Para a determinação de $\mathrm{pH}$, homogeneizou-se bem a amostras, transferiu-se para recipiente adequado, medindo-se o pH com auxílio de potenciômetro previamente calibrado com soluções tampões de padronização, dotadas de pH 4, 0 e 7,09.

A determinação da densidade foi realizada conforme descrito na Farmacopéia Brasileira ${ }^{9}$, utilizando-se picnômetro previamente calibrado. Para a realização das análises a temperatura foi padronizada em 20 oC. 0 quociente entre a massa da amostra líquida e a massa da água, ambas a $20^{\circ} \mathrm{C}$, é a densidade relativa.

Saúde (Santa Maria), v.38, n.1, p. 139-149, 2012 
Procedeu-se à análise da viscosidade aparente conforme descrito na Farmacopéia Brasileira ${ }^{9}$, empregando-se viscosímetro rotacional digital Brookfield, modelo RV DV -1+, na velocidade de $50 \mathrm{rpm}$. Para a realização das análises, a temperatura das amostras foi padronizada em $25 \mathrm{OC}$, obedecendo-se o tempo de 1 minuto para 0 ajuste do fator de velocidade.

\section{Resultados e discussão}

No teste de identificação do cetoconazol, os tempos de retenção dos picos principais obtidos dos cromatogramas das soluções amostra, corresponderam àquele do pico principal da solução padrão, conforme exemplificado nas Figuras 1 e 2.
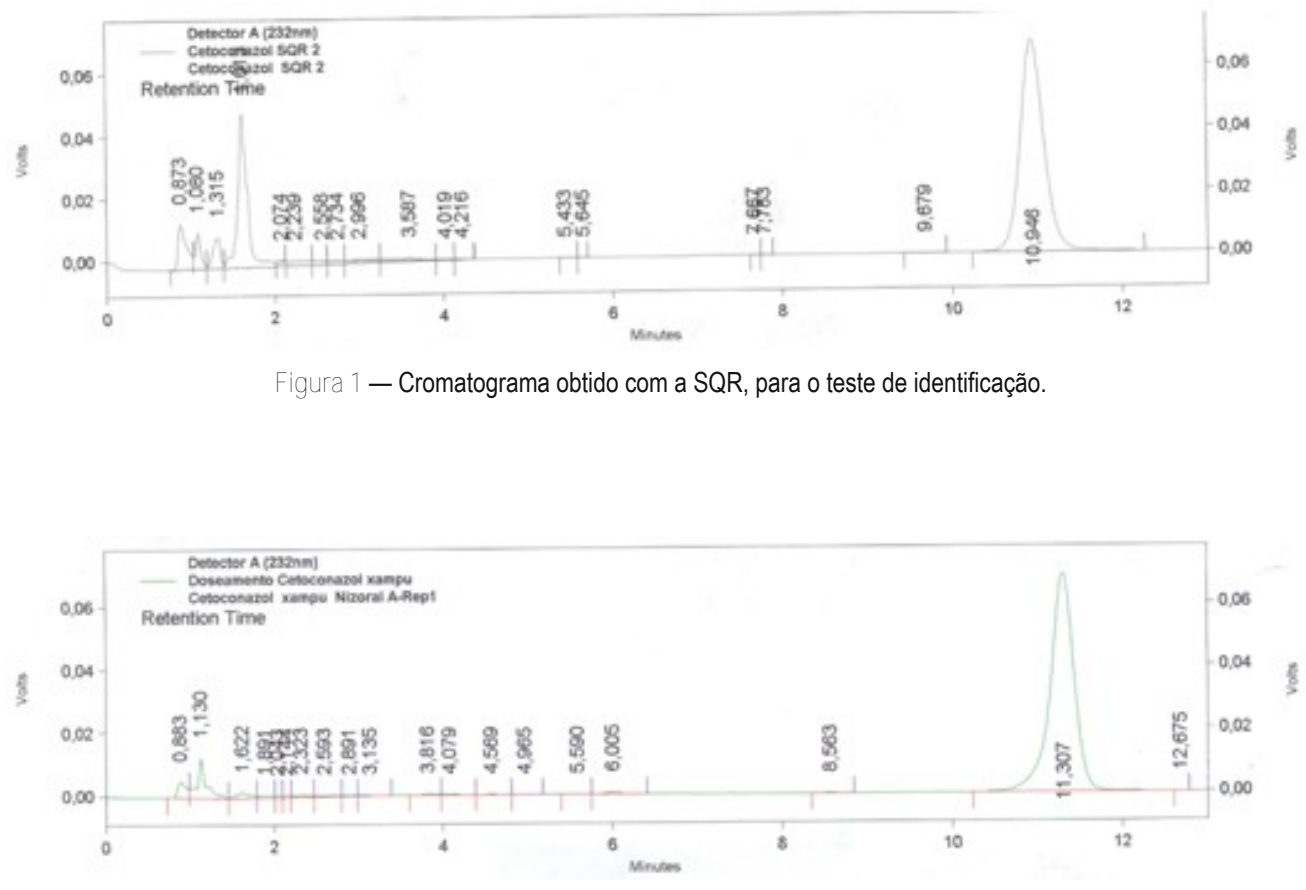

Figura 2 - Cromatograma obtido com uma das amostras, para o teste de identificação.

A determinação de volume visa informar a constancia do envase por unidade do lote. Os limites de variação permitidos são calculados de acordo com o volume declarado em cada produto. Para que as amostras sejam aprovadas o valor médio obtido entre as unidades testadas não deverá ser inferior ao declarado e nenhuma unidade poderá ultrapassar 0 desvio máximo, que nesse caso é de $1,5 \%$.

O medicamento teste e as amostras manipuladas apresentaram volume médio superior ao declarado, não ultrapassando os limites da Farmacopéia Brasileira ${ }^{9}$, conforme descrito na Tabela 1. 
Tabela 1 - Valores obtidos na determinação de volume das formulações 1 e 2 manipuladas e medicamentos de referencia, genérico e similar.

\begin{tabular}{cccccc}
\hline Frasco & Formulação 1 & Formulação 2 & $\begin{array}{c}\text { Medicamento } \\
\text { referência }\end{array}$ & $\begin{array}{c}\text { Medicamento } \\
\text { genérico }\end{array}$ & $\begin{array}{c}\text { Medicamento } \\
\text { similar }\end{array}$ \\
\hline 2 & 99 & 98 & 101 & 104,47 & 100,30 \\
3 & 100,05 & 100,41 & 100,54 & 101,41 & 100,28 \\
Média & 100,23 & 100,53 & 101,48 & 101,49 & 99,84 \\
\hline DP & 100,09 & 100,66 & 100,82 & 101,46 & 100,14 \\
\hline DPR\% & 0,13 & 0,33 & 0,57 & 0,04 & 0,26 \\
\hline LImite minımo & 0,13 & 0,33 & 0,56 & 0,04 & 0,26 \\
\hline Limite máximo & 101,50 & 101,50 & 101,50 & 101,50 & 101,50 \\
\hline & 98,50 & 98,50 & 98,50 & 98,50 & 98,50 \\
\hline
\end{tabular}

Os limites especificados na Farmacopéia Brasileira ${ }^{9}$ para esse fármaco no doseamento são de, no mínimo, $90 \%$ e, no máximo, $110 \%$ da quantidade declarada de cetoconazol. Todos os produtos analisados cumpriram com a especificação, conforme demonstrado na Tabela 2. Tabela 2 - Resultados obtidos nos doseamentos dos xampus de cetoconazol a $2 \%$.

\begin{tabular}{ccc}
\hline Concentração $(\mathrm{g} / \mathrm{mL})$ & Teor $(\%)+$ & Desvio Padrão \\
\hline Referência & $100,05+$ & 0,48 \\
Genérico & $101,01+$ & 3,27 \\
Similar & $104,70+$ & 0,04 \\
Formulação 1 & $99,17+$ & 0,15 \\
Formulação 2 & $101,68+$ & 1,04 \\
\hline
\end{tabular}

Analisando os resultados dos testes de estabilidade, em relação ás alterações de cor e odor das amostras, foi verificado que as formulações comerciais praticamente não sofreram alterações de cor e odor quando armazenadas a $4^{\circ} \mathrm{C}$ e $25{ }^{\circ} \mathrm{C}$. Os medicamentos manipulados mantiveram-se estáveis a temperatura de $4^{\circ} \mathrm{C}$, mas quando armazenadas a 25 ${ }^{\circ} \mathrm{C}$ começaram a sofrer pequenas alterações de cor, próximo aos 180 dias. As formulações armazenadas a $40{ }^{\circ} \mathrm{C}+70 \%$ UR sofreram grandes alterações de cor, a partir dos 30 dias, principalmente as manipuladas.

Os produtos comerciais possuíam colorações laranja-avermelhadas e as formulações manipuladas não tinham corantes. Foi possível observar, durante 0 estudo, que 0 cetoconazol confere uma cor laranja ao xampu que, com o decorrer do tempo e aumento da temperatura, ocasiona a coloração vermelha de todo o produto. Essa alteração de cor deve estar relacionada à perda de estabilidade ou início da degradação do produto (Figura 3). 

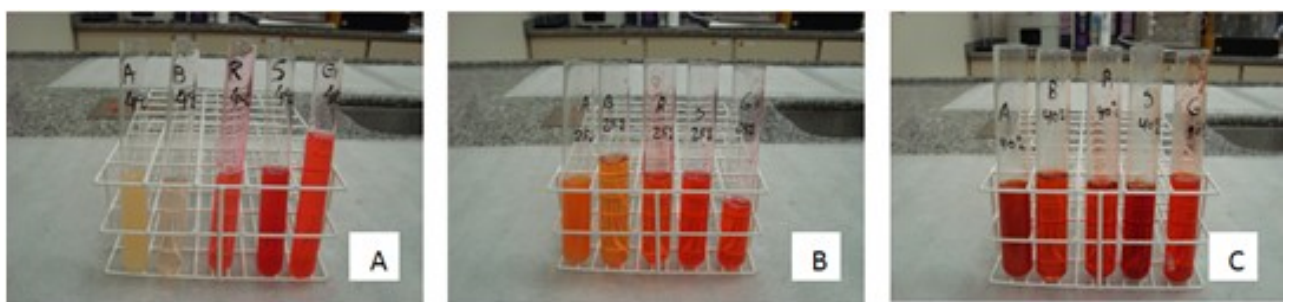

Figura 3 - Imagens das amostras aos 180 dias de análise, nas temperaturas de 4 oC (A); 25 oC (B), e 40 oC + 70\% UR (C).

A segurança biológica in vivo (teste de irritação ocular) e in vitro (teste de citotoxicidade) do xampu de cetoconazol degradado sob ação de radiação UV-C $(254 \mathrm{~nm})$ foi avaliada por Staub e colaboradores (2007). Neste estudo, o cetoconazol das amostras foi quantificado por CLAE e ensaio microbiológico. Ocorreram alterações na substancia em presença de luz, o que foi demonstrado pela presença de picos secundários no cromatograma, além da diminuição da atividade antifúngica do composto e uma intensa mudança de coloração do produto. Entretanto, nos ensaios de segurança biológica não foram demonstradas alterações entre o produto degradado e não degradado ${ }^{11}$.

Neste estudo a mudança de coloração do xampu foi relacionada a degradação do produto evidenciada no cromatograma. Em nosso trabalho, apesar do aumento na intensidade da coloração no teste de estabilidade, não foram encontrados produtos de degradação no cromatograma e houve uma pequena diminuição no teor do fármaco com o passar do tempo. Com isso verifica-se que um estudo mais aprofundado deve ser realizado a fim de determinar quais os compostos formados a partir da degradação do cetoconazol, além da possibilidade destes produtos estarem co-eluindo com o fármaco durante a análise cromatográfica.

Em condições ideais, o pH do cabelo está entre 4 e 5.0 uso de determinados tipos de xampus pode levar a mudanças no $\mathrm{pH}$ do mesmo e promover alterações na estrutura capilar. Recomenda-se que os xampus de uso diário tenham o pH a faixa de 5 a 7 , se o pH for maior abrirá as cutículas em maior profundidade, como é o caso dos xampus anti-resíduos. Um xampu neutro é de fato melhor para os cabelos que um alcalino, mas o ideal é que ele seja levemente ácido ${ }^{12}$. Não existe referência para o pH de xampus de cetoconazol, mas sabe-se que se for levemente ácido a ação antifúngica e antibacteriana é mais eficiente, servindo ainda como proteção ao couro cabeludo.

Nos testes realizados, nenhuma das formulações apresentou diferenças significativas $(p<0,5)$ de $\mathrm{pH}$ no decorrer dos 180 dias, ficando todos entre a faixa de 5 a 7 , que pode ser considerada ideal para xampus, conforme representado graficamente na Figura 4. 


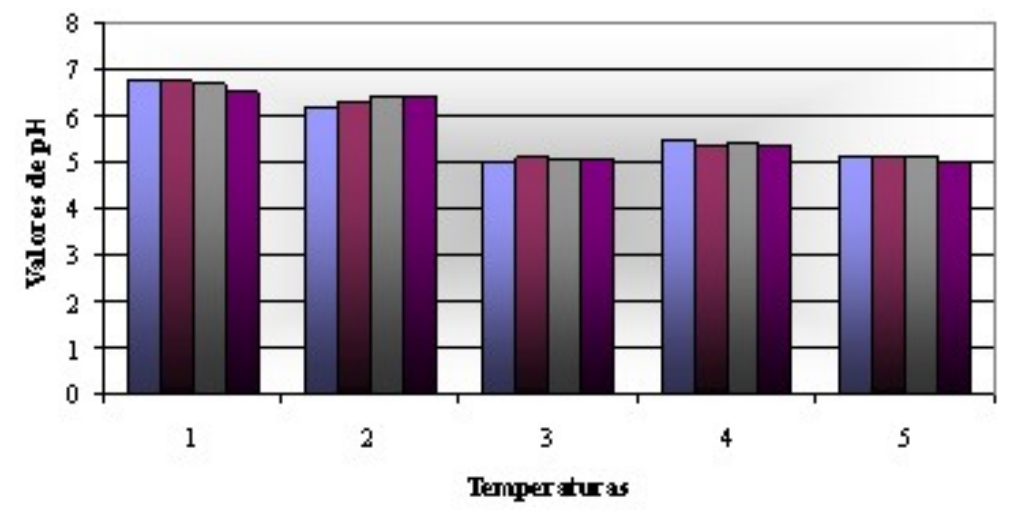

$\square$ Inicial $\square 4^{\circ} \mathrm{C} \square 25^{\circ} \mathrm{C} \square 40^{\circ} \mathrm{C}+70 \% \mathrm{UR}$

Figura 4 - Representação gráfica da variação do pH para cada temperatura, sendo (1) medicamento referência, (2) medicamento genérico; (3) medicamento similar; (4) formulação 1 e (5) formulação 2.

Após análise estatística dos valores relativos à densidade, no tempo zero (To), 30, $90 \mathrm{e}$ 180 dias das amostras, foi verificado que não houve diferenças significativas $(p>0,5)$ na densidade em nenhuma das formulações.

A viscosidade de um fluido pode ser descrita simplesmente como sua resistência ao fluxo ou movimento. Em um xampu, normalmente, corrige-se o pH até a faixa estabelecida, depois se procede a correção da viscosidade, com a adição de cloreto de sódio ${ }^{13}$. As medidas reológicas provêm informações sobre a estabilidade física e a consistência do produto, indicando se os produtos mantêm suas propriedades intrínsecas e de escoamento durante 0 tempo que permanecem em prateleira ${ }^{14}$.

A Figura 5 demonstra as variações das viscosidades dos produtos, quando estocadas nas temperaturas de $4 \mathrm{OC} ; 25 \mathrm{OC}$, e $40 \mathrm{OC}+70 \%$ UR, durante seis meses.
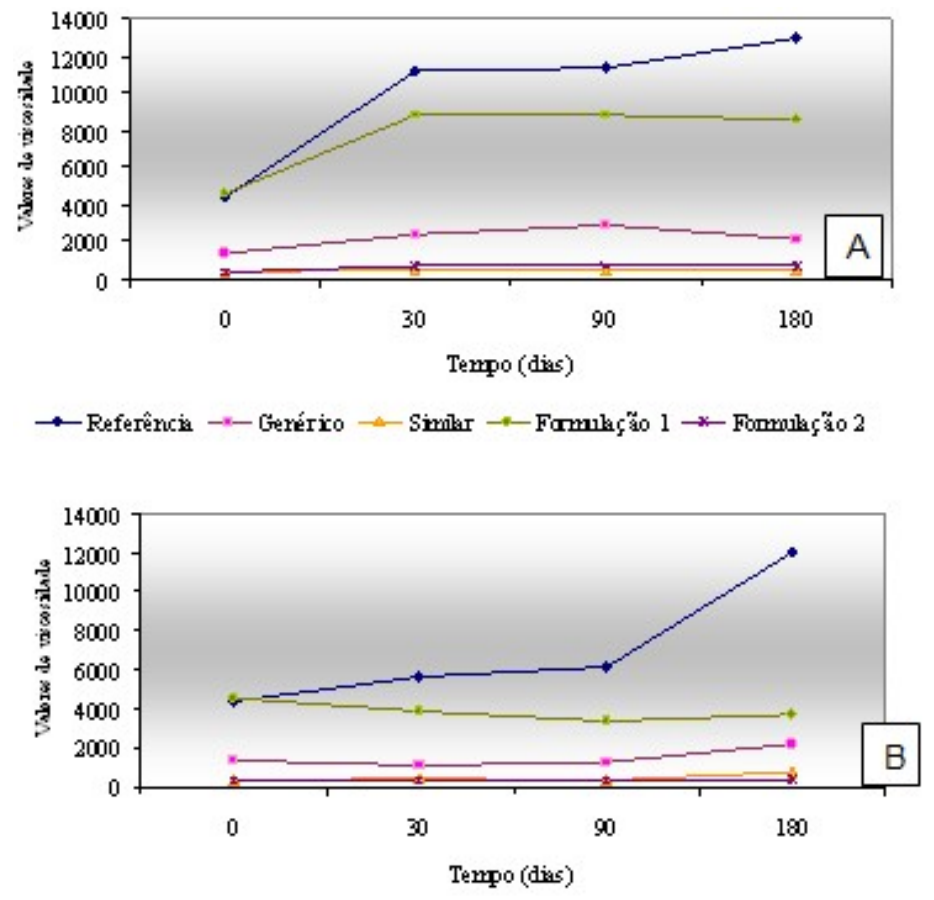

Saúde (Santa Maria), v.38, n.1, p. 139-149, 2012 Gindri, L. L.; et al. 


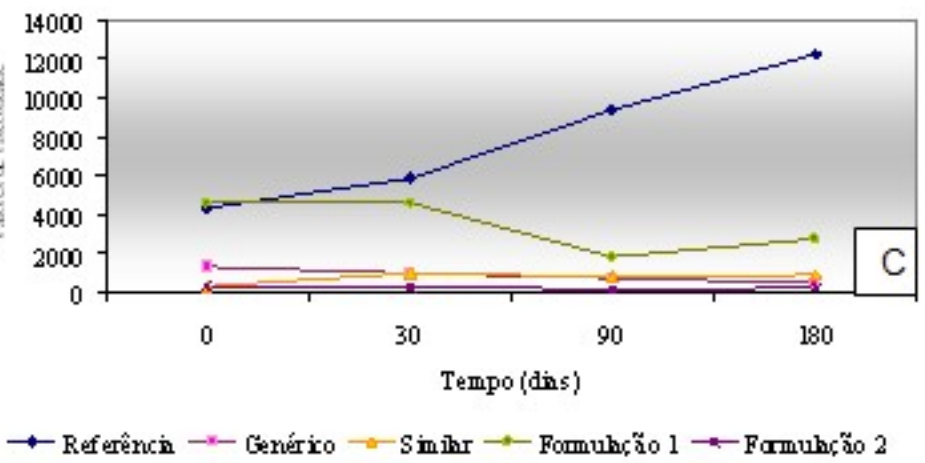

Figura 5 - Representação gráfica da variação da viscosidade, na temperatura de 4 oC (A), 25 oC (B) e 40oC + 70\% UR (C), durante o tempo zero, 30, 90 e 120 dias.

O medicamento referência teve um aumento da viscosidade, em todas as temperaturas, com o passar do tempo. Quando comparado estatisticamente, por ANOVA, não apresentou diferença significativa $(p<0,05)$ entre as condições de armazenamento, mas apresentou diferença significativa $(p>0,05)$ entre os tempos em que foi analisado.

No tempo zero, podemos observar que o medicamento similar e a formulação 2 tinham uma viscosidade extremamente baixa, o que não acontecia com as demais formulações. Esses dois produtos foram os que menos apresentaram alteração na viscosidade no decorrer do estudo, sendo que a formulação 2 somente apresentou diferença significativa $(p<0,05)$ quando comparamos as temperaturas de estocagem.

O medicamento genérico quando comparado estatisticamente, por ANOVA, apresentou diferença significativa $(p<0,05)$ entre as condições de armazenamento, mas não apresentou diferença significativa $(p>0,05)$ entre os tempos em que foi analisado. 0 mesmo aconteceu com a formulação 1.

Todos os medicamentos apresentaram um aumento de viscosidade quando estocados a $4 \mathrm{oC}$, resultado característico de produtos mantidos a baixa temperatura, porém no momento em que foram avaliados já estavam a temperatura de 25 oC.

Como as amostras tiveram comportamentos diferenciados em relação a viscosidade, não conseguimos estabelecer um parâmetro ideal. Esse aspecto também foi observado por Quadros, 2007, quando avaliou a viscosidade de diferentes xampus ${ }^{15}$.

Segundo a RE no 1, de 29 de julho de 2005, para fins de prazo de validade provisório de 24 meses será aprovado o relatório de estabilidade acelerado ou de longa duração de 12 meses que apresentar variação menor ou igual a $5,0 \%$ do valor de análise da liberação do lote, mantidas as demais especificações. Somente a formulação 2 apresentou queda de teor superior a $5 \%$, sendo que todos os demais produtos, já teriam garantido o prazo de validade de 2 anos para a comercialização ${ }^{10}$.

0 estudo de estabilidade foi conduzido em três temperaturas, pois caso os produtos apresentassem uma degradação de, no mínimo $30 \%$ do ativo, seria possível calcular o prazo de validade pelo método de Arrhenius ${ }^{16}$.

Segundo Grimm (2001) outra forma de se calcular o prazo de validade de produtos

Saúde (Santa Maria), v.38, n.1, p. 139-149, 2012. Estudo da estabilidade acelerada de formulaçōes contendo cetoconazol xampu a $2 \%$ farmacêuticos é plotar em um gráfico, tempo $(x)$ VS. teor (y), e calcular o limite de confiança de $95 \%$. O prazo de validade é obtido, extrapolando-se o eixo de $x$ até encontrar a linha do limite de confiança, quando a mesma toca o limite inferior de teor permitido para cada produto $^{17}$, no caso do cetoconazol, $90 \%$ de ativo, conforme demonstrado graficamente na Figura 6. 

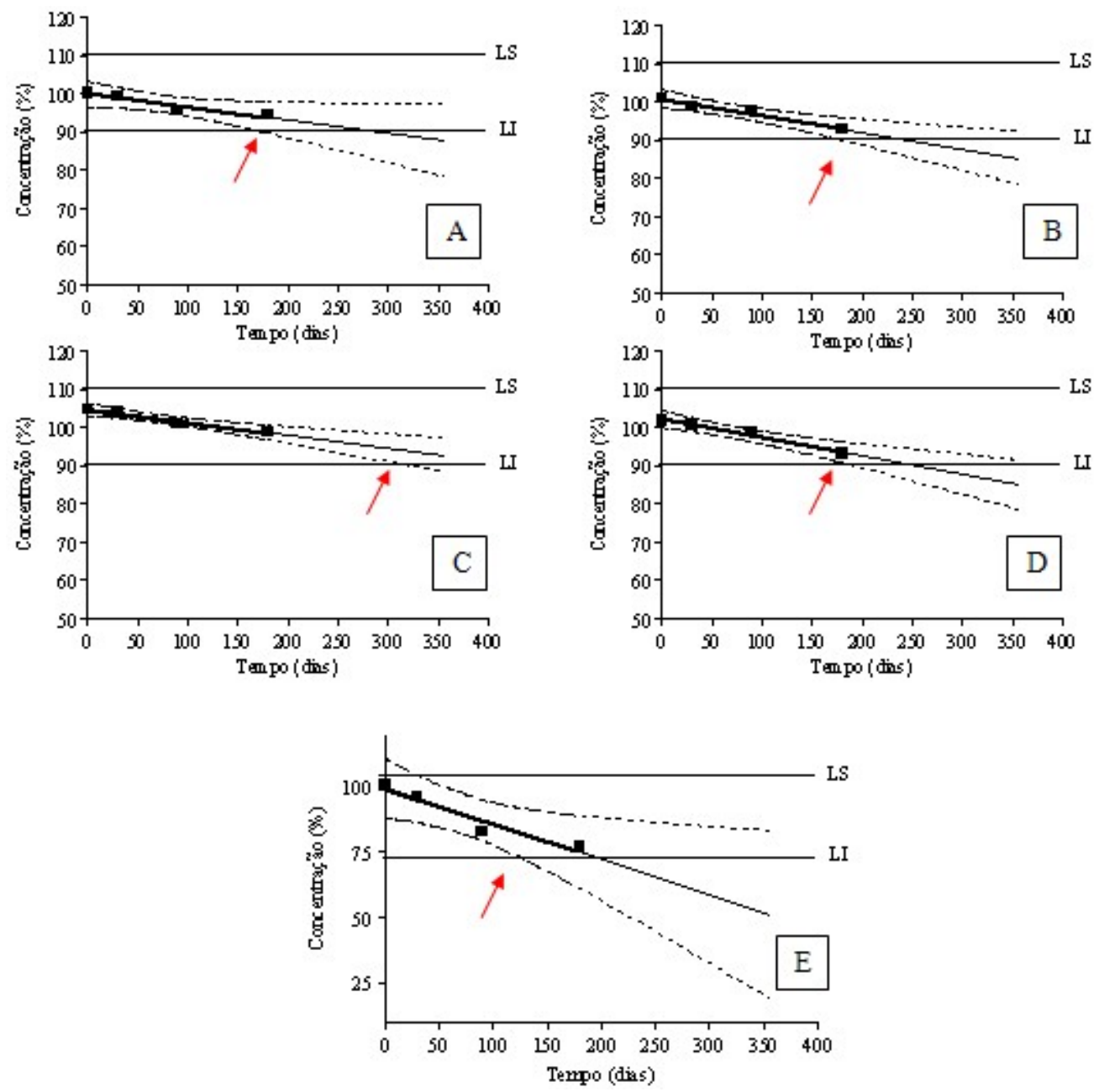

Figura 6 - Representação gráfica da determinação do prazo de validade para o medicamento referência $(A)$, medicamento genérico (B), medicamento similar (C), formulação 1 (D), formulação 2 (E), utilizando-se os valores da temperatura de 40 oC +/$70 \%$ UR.

Quando as amostras são avaliadas por esse parâmetro, o prazo de validade do medicamento referência ficou em 182 dias, do genérico em 173 dias e do similar em 317 dias. Cabe ressaltar que o produto similar tem um prazo validade maior, pois seu teor inicial era de $104 \%$ e, de todas as outras formulações, próximo a 100\%. As formulações 1 e 2 tiveram prazos de validade de 180 dias e 125 dias, respectivamente.

\section{Conclusões}

As amostras analisadas foram aprovadas no teste de identificação, volume médio e doseamento, conforme especificações da Farmacopéia Brasileira, 2009.

As formulações comerciais praticamente não sofreram alterações de cor e odor quando armazenadas a $4{ }^{\circ} \mathrm{C}$ e $25^{\circ} \mathrm{C}$. Os medicamentos manipulados mantiveram-se estáveis a temperatura de $4{ }^{\circ} \mathrm{C}$, mas quando armazenadas a $25{ }^{\circ} \mathrm{C}$ começaram a sofrer pequenas Saúde (Santa Maria), v.38, n.1, p. 139-149, 2012. Gindri, L. L.; et al. alterações de cor próximo aos 180 dias. As formulações armazenadas a $40{ }^{\circ} \mathrm{C}+70 \%$ UR 
sofreram grandes alterações de cor, a partir dos 30 dias, principalmente as manipuladas. Nos medicamentos manipulados as diferenças nas colorações foram melhor observadas devido ao fato destes não apresentarem colorações iniciais.

Não houve alterações consideráveis de $\mathrm{pH}$ e densidade, para todas as amostras analisadas, nas temperaturas de $4 \mathrm{OC} ; 25 \mathrm{OC}$, e $40 \mathrm{OC}+70 \%$ UR, durante seis meses.

Os xampus mostraram comportamento diferente, em relação à viscosidade, com exceção da temperatura de $4 \mathrm{oC}$, em que todas as formulações aumentaram o valor, sendo impossível determinar a faixa ideal de viscosidade. 0 medicamento referência teve um aumento da viscosidade, em todas as temperaturas, com o passar do tempo; o medicamento similar e a formulação 2, foram os que apresentaram menor viscosidade no inicio do estudo e também foram os que apresentaram menor variação em função do tempo. 0 medicamento genérico e a formulação 1 apresentaram diminuição da viscosidade, a temperatura de $40 \mathrm{oC}$ $+70 \%$ UR.

Os medicamentos comerciais e a formulação 1 mostraram boa estabilidade, sendo que 0 teor não foi inferior a 90\%, após 180 dias de análise, a temperatura de 40 oC + 70\% UR. Com esse resultado, frente ao setor de registro da ANVISA, seria possível conseguir um prazo de validade provisório de 24 meses. Já a formulação 2, que era a única com base perolada, mostrou-se menos estável ao estudo de estabilidade acelerada, indicando não ser a formulação ideal para cetoconazol xampu.

O medicamento similar e a formulação 2 foram os que apresentaram maior perda de teor e os que conferiram coloração mais avermelhada aos xampus. A formação ou aumento da intensidade da coloração das formulações contendo cetoconazol deve ser melhor investigada, a fim de confirmar se esta alteração ocorre devido a degradação do principio ativo e definir se há diminuição na atividade antifúngica do produto.

Quando avaliamos as amostras pelo método de regressão linear, observamos que quase todas as formulações vão apresentar prazo de validade semelhante, com exceção do medicamento similar que, apesar de apresentar a maior queda de teor, tinha no inicio do estudo $104 \%$ de ativo, o que não acontece com os demais produtos.

\section{Referências}

1. GOODMAN \& GILMAN, As bases farmacológicas da terapêutica. 9.ed. Rio de Janeiro: McGrw-Hill interamericana, S.A. DE C.V, 2006, p.1821

2. FUCHS, FD. WANNMACHER, L. FERREIRA, MBC. Farmacologia clínica, fundamentos da terapia racional. 3.ed. Rio de Janeiro: Guanabara Koogan S.A, 2006, p.431-444

3. KATZUNG, BG. Farmacologia básica e clínica. 8.ed. Rio de Janeiro: Guanabara Koogan, 2002. 1046 p.

4. ANTONIO, MECO. Permeação Cutânea IN VITRO como ferramenta auxiliar para o estudo de formulações semi-sólidas de Cetoconazol para aplicações tópicas. Dissertação (mestrado) - Universidade Federal do Paraná, Setor de Ciências da Saúde, Programa de Pós-Graduação em Ciências Farmacêuticas. Defesa: Curitiba, 2007 Biblioteca Digital de Teses e Dissertações, disponível em: http://hdl.handle.net/1884/12097, acessado em: 15/10/2008

Saúde (Santa Maria), v.38, n.1, p. 139-149, 2012. 
6. STORPIRTIS, S. CONSIGLIERI, VO. Biodisponibilidade e bioequivalência de medicamentos, aspectos fundamentais para o planejamento e execução de estudos. Revista da faculdade de farmácia e bioquímica da Universidade de São Paulo. 1995; 31(2): 63.

7. GIL, ES. Controle físico-químico de qualidade de medicamentos. 2.ed. São Paulo: Pharmabooks 2007. $485 \mathrm{p}$.

8. BRASIL, Formulário Nacional / Ministério da Saúde, Agência Nacional de Vigilância Sanitária. - Brasília : Ministério da Saúde, 2005

9. FARMACOPÉIA Brasileira. 4.ed. São Paulo: Atheneu, 1988.

10. BRASIL, Ministério da Saúde, Secretaria Nacional de Vigilância Sanitária. Resolução RE no1. Diário Oficial da Repúplica Federativa do Brasil, 29 jul. 2005. Guia para a realização de estudos de estabilidade, 2005.

11. STAUB, I; CRUZ, AS; PINTO,TJA; SCHAPOVAL, EES; BERGOLD, AM. Determinação da segurança biológica do xampu de cetoconazol: teste de irritação ocular e avaliação do potencial de citotoxicidade in vitro. Revista Brasileira de Ciências Farmacêuticas. 2007; 43(2):301-307.

12. BARBOSA, AB.; SILVA, RR. Xampus. Química Nova na Escola. 1995; 2: 3-6

13. AULTON, ME. Delineamento de formas farmacêuticas, parte 1, 2 ed.; 2005. 677 p.

14. TADROS, T. Application of rheology for assessment and prediction of the long-tern physical stability of emulsions. Advances in Colloid and Interface Science. 2004; 108-109: 227-258

15. QUADROS, L. Avaliação da viscosidade em diferentes tipos de xampus. 2007. 32f. Trabalho final de graduação em Farmácia, Centro Universitário Franciscano, Santa Maria, RS, 2007.

16. NUDELMAN, NS. Estabilidade de medicamentos. Buenos Aires: El Ateneo, 1975, p.1-4

17. GRIMM, W. Estabilidade de fármacos e medicamentos. Sindusfarma, São Paulo, 2001.

\section{Amanda Leitão Gindri}

Endereço para correspondência - Rua Benjamin Constant, $n^{\circ} 930$, apto 114, Centro, Santa Maria, RS. CEP: $97.050-020$

E-mail: amandagindri@terra.com.br

Currículo lattes: http://buscatextual.cnpq.br/buscatextual/visualizacv.do?id=K4476693U8

Recebido em 14 de janeiro de 2011.

Aprovado em 24 de agosto de 2012.

Saúde (Santa Maria), v.38, n.1, p. 139-149, 2012 